\title{
Kindliches Wohlbefinden in unterschiedlichen Familienformen: ein Fokus auf komplexe Stieffamilien
}

\author{
Christine Entleitner-Phleps • Sabine Walper
}

Online publiziert: 27. März 2020

(C) Der/die Autor(en) 2020

Zusammenfassung Im Kontext wachsender Scheidungsraten wurden dem kindlichen Wohlbefinden in Stief- und alleinerziehenden Familien in den letzten Jahrzehnten viel Aufmerksamkeit geschenkt. Die Heterogenität von Stieffamilien wurde dabei allerdings weitgehend vernachlässigt. Basierend auf Daten des repräsentativen Surveys „Aufwachsen in Deutschland: Alltagswelten“ $(2009, N=2144)$ vergleicht die vorliegende Studie das selbst berichtete Wohlbefinden (SDQ) von Kindern (9-12 Jahre) in Kernfamilien, Stiefvaterfamilien, komplexen Stieffamilien mit mindestens einem gemeinsamen Kind und Familien mit einer alleinerziehenden Mutter. Die Ergebnisse zeigen, dass Kinder, die mit einer alleinerziehenden Mutter oder in einer komplexen Stieffamilie leben, Nachteile hinsichtlich ihres Wohlbefindens aufweisen. Die Kinder geben ein schlechteres Familienklima und mehr Streit in der Familie an als Kinder in Kernfamilien. Bei Kindern aus (einfachen) Stiefvaterfamilien konnten keine Nachteile nachgewiesen werden. Die Interpretation der Ergebnisse verweist auf unterschiedliche Risiken und Herausforderungen, mit denen einerseits Alleinerziehende, andererseits aber auch komplexere Stieffamilienformen konfrontiert sind.

Schlüsselwörter Kindliches Wohlbefinden · Scheidung · Familienbeziehungen · SDQ $\cdot$ Stieffamilientypen 


\title{
Child well-being in diverse family structures: a focus on complex stepfamilies
}

\begin{abstract}
In the context of increasing divorce rates, much attention has been paid to children's well-being in separated single-parent and stepfamilies. However, the heterogeneity and variations in complexity in stepfamilies have long been neglected. Based on data from the representative survey "Growing up in Germany" (2009, $N=2144$ ), the present study compares the self-reported psychological well-being of children (9-12 years), assessed with the Strenghts and Difficulties Questionaire (SDQ), in nuclear, single-mother, simple stepfather families, and complex stepfamilies (with at least one joint child). The findings suggest disadvantages for children's well-being in single-mother and complex stepfamilies which can largely be accounted for by the lower level of family cohesion and increased family conflict reported by these children when compared to children in nuclear families. No disadvantages were found for children in simple stepfather-families. The findings are interpreted with respect to the diverse risks and challenges involved in managing not only single parenthood but also increased family complexity.
\end{abstract}

Keywords Child well-being $\cdot$ Divorce $\cdot$ Family relations $\cdot$ SDQ $\cdot$ Stepfamily types

\section{Einleitung}

In den letzten Jahrzehnten hat ein tiefgreifender Wandel in der Struktur und Ausgestaltung des Familienlebens stattgefunden, der die Bedingungen des Aufwachsens von Kindern und Jugendlichen einschließt (Jurczyk und Klinkhardt 2014, S. 17 ff.). Angesichts hoher Scheidungs- und Trennungsraten lebt ein steigender Anteil der Kinder und Jugendlichen zumindest zeitweise mit nur einem Elternteil, erlebt vielfach aber auch die Gründung einer Stieffamilie, die das Zusammenleben mit einem neuen sozialen Elternteil umfasst. Allein in Deutschland waren 2017123.563 minderjährige Kinder mit einer Scheidung der Eltern konfrontiert (Statistisches Bundesamt 2018). Die steigende Quote nicht-ehelicher Geburten - im Jahr 2017 betraf dies 34,7\% der Geburten (Statistisches Bundesamt 2019, S. 3) - befördert diesen Trend, da nichteheliche Partnerschaften sich als weniger stabil erweisen (Bastin 2016).

Wie sich eine Trennung der Eltern und in der Folge neue Partnerschaften auf das Wohlbefinden von Kindern auswirken, ist in der internationalen Forschung Gegenstand vieler Untersuchungen (vgl. z. B. Amato 1993, 2010, 2014; Coleman et al. 2000). Meta-Analysen (z.B. Amato 2014) schlussfolgern, dass Kinder aus Trennungsfamilien vielfach Nachteile hinsichtlich ihres Wohlergehens aufweisen, wobei diese Nachteile jedoch insgesamt eher schwach ausfallen und beträchtliche Heterogenität bergen. Vielfältige Faktoren, wie die ökonomische Situation des Haushalts, das elterliche Konfliktniveau sowie Probleme im Erziehungsverhalten und in der elterlichen Zusammenarbeit in der Erziehung beeinflussen die Trennungsbewältigung und können teilweise die Nachteile von Kindern aus Trennungsfamilien erklären (Amato 2000). 
Geht der hauptbetreuende Elternteil eine neue Partnerschaft ein und entschließen sich die Partner nach der Stieffamiliengründung ein weiteres gemeinsames Kind zu bekommen, wird die neue Familiensituation zunehmend komplex, nicht nur hinsichtlich verschiedener Abstammungslinien, sondern auch hinsichtlich der Organisation und der Ausgestaltung des Familienlebens, das auch Kontakte der Trennungskinder zum getrennt lebenden Elternteil umfasst. Komplexe Stieffamilien stehen damit vielfältigen Herausforderungen gegenüber.

In empirischen Untersuchungen kann die Heterogenität der Familienstruktur auch aufgrund fehlender Daten - häufig nicht differenziert abgebildet werden. Insbesondere die Diversität von Stieffamilien in Bezug auf die Geschwisterkonstellationen wird kaum berücksichtigt. Untersuchungen, welche das Wohlergehen von Kindern in Stieffamilien untersuchen, sind sich zudem nicht einig hinsichtlich der Vor- und Nachteile dieser Familienkonstellationen (z. B. Coleman et al. 2000; McMunn et al. 2001; O’Connor et al. 2001; Sweeney 2007). Zumindest ein Teil dieser Uneinigkeit resultiert aus der Tatsache, dass eine differenzierte Analyse dieser diversen Stieffamilienformen oft nicht möglich ist. Einige Studien stufen Stiefkinder und gemeinsame Kinder, die in einer Stieffamilie aufwachsen, als Kinder derselben Familienstruktur ein oder klassifizieren gar Stieffamilien, in denen ein gemeinsames Kind lebt, als Kernfamilien, da zumeist Informationen über alle im Haushalt lebenden Kindern fehlen (Gennetian 2005, S. 431).

Die vorliegende Studie geht deshalb der Frage nach, welche Rolle die Familienform, in der die Kinder aufwachsen für kindliches Wohlbefinden spielt. Dabei differenziert die vorliegende Studie zwischen verschiedenen (Stief-)Familienformen und vergleicht (1) Stieffamilien, in denen alle Kinder mit einem gemeinsamen leiblichen und einem sozialen Elternteil in einem Haushalt leben (einfache Stieffamilien), (2) Stieffamilie mit mindestens einem gemeinsamen Kind (komplexe Stieffamilien), (3) Familien mit einem Elternteil (Alleinerziehende) und (4) Kernfamilien mit zwei leiblichen Eltern.

\section{Das Wohlergehen von Kindern und Jugendlichen in komplexen Stieffamilien: Forschungsüberblick}

Das Wohlergehen von Kindern (child well-being) steht schon seit den 1960er Jahren im Mittelpunkt der Scheidungsfolgenforschung (Amato 1993) und hat auch in der Sozialberichterstattung zunehmend an Bedeutung gewonnen. Hierbei werden unterschiedliche Konzepte und Zugänge zugrunde gelegt. Während einige Arbeiten ausschließlich objektive Faktoren wie Erkrankungen oder die ökonomische Situation der Kinder in den Blick nehmen (von Rueden et al. 2006), sind in den letzten Jahren vermehrt auch subjektive Faktoren wie Zufriedenheit in verschiedenen Lebensbereichen, Glücklich-Sein aber auch psychisches Wohlbefinden berücksichtigt worden (Ben-Arieh und Frønes 2011). Hierbei ist es entscheidend, die Kinder und Jugendlichen selbst zu Wort kommen zu lassen und sie direkt nach ihrem Wohlbefinden zu fragen (Hurrelmann et al. 2013; Albert et al. 2019), da ihr Erleben Außenstehenden und selbst den Eltern nur in begrenztem Maße zugänglich ist. Wir knüpfen an diese Arbeiten an, indem wir die Perspektive der Kinder in den Mittelpunkt stellen und 
deren subjektives Wohlbefinden in Bezug auf die seelische Gesundheit fokussieren. Subjektives Wohlbefinden ist hierbei als Teilaspekt des Wohlergehens von Kindern zu verstehen. Wenn wir im Folgenden einen Überblick über die Forschung zu kindlichem Wohlergehen in komplexen Familienstrukturen geben, gehen wir nicht nur auf subjektives Wohlbefinden, sondern auch weitere Bereiche des Wohlergehens auch aus Perspektiven der Eltern - ein.

\subsection{Kindliches Wohlbefinden im Kontext komplexer Familienstrukturen}

Komplexe Familienstrukturen entstehen durch unterschiedliche Kindschaftsverhältnisse von Geschwistern innerhalb eines Haushalts. Wenngleich komplexe Familienverhältnisse nicht ausschließlich auf Stieffamilien beschränkt sind - so können auch in Alleinerziehenden-Familien mehrere Kinder aus unterschiedlichen Partnerschaften des/der Alleinerziehenden zusammenleben (Stewart 2006) - wird in diesem Beitrag der Fokus auf die Komplexität von Stieffamilien mit gemeinsamen Kindern gelegt.

Komplexe Stieffamilien entstehen, wenn in einer Stieffamilie ein gemeinsames Kind der Partner geboren wird (Ganong und Coleman 2004, S. 86f.). Meist geht dieser Situation eine einfache Stieffamilie voraus, in der lediglich einer der Partner bereits Kinder aus einer früheren Partnerschaft hat. Seltener bringen beide Partner Kinder aus früheren Partnerschaften in den gemeinsamen Haushalt der Stieffamilie ein, und bekommen zusätzlich ein gemeinsames Kind (für eine ausführliche Darstellung verschiedener Stieffamilientypen siehe z. B. Walper et al. 2016, S. $215 \mathrm{ff}$.). In beiden Fällen bedeutet die Geburt eines gemeinsamen Kindes, dass der soziale Elternteil nicht mehr „nur“ aufgrund der Partnerschaft mit dem leiblichen Elternteil in das Familiengefüge eingebunden ist, sondern nun auch die Rolle als leiblicher Elternteil eines gemeinsamen Kindes einnimmt.

Einige Studien beschäftigen sich mit der Frage, wie die Geburt eines gemeinsamen Kindes das Familienleben und insbesondere das Engagement des sozialen Elternteils mit den Stiefkindern beeinflusst. Die Befunde legen nahe, dass ein gemeinsames Kind weder mit einer Verschlechterung des Engagements für die Stiefkinder einhergeht, noch dass dieses Ereignis die Rolle des sozialen Elternteils stärkt (Hofferth und Anderson 2003; Stewart 2005). Allerdings können geringe sozioökonomische Ressourcen die Situation erschweren. Komplexe Stieffamilien gründen sich häufig infolge früher Elternschaft in instabilen Partnerschaften, was vermehrt auf Eltern mit geringeren Bildungsressourcen zutrifft (Furstenberg 2014, S. 21 f.). Vor diesem Hintergrund überrascht es nicht, dass eine Studie von Brown et al. (2015) vermehrte ökonomische Nachteile - als ein Aspekt des kindlichen Wohlergehens - für Kinder aufzeigt, die in komplexen Familienstrukturen (mit Halb- und Stiefgeschwistern) aufwachsen.

Nachteile von Kindern in einer komplexen Familienstruktur betreffen allerdings nicht nur die ökonomischen Ressourcen. Eine der wenigen Untersuchungen, die Jugendliche aus unterschiedlich komplexen Familienkonstellationen verglich und hierbei deren Angaben zu verschiedenen Aspekten des Wohlergehens wie deren schulischen Leistungen, Fehlverhalten in der Schule, Delinquenz und Depressivität in den Blick nahm, erbrachte für Jugendliche in komplexen Stieffamilien schlechtere 
Werte als für Jugendliche in Kernfamilien (Halpern-Meekin und Tach 2008). Interessanterweise galten diese Ergebnisse nicht nur für Stiefkinder, sondern auch für gemeinsame Kinder in komplexen Stieffamilien, die keine elterliche Trennung erlebt hatten, sondern seit jeher mit beiden leiblichen Eltern in einem Haushalt lebten. Obwohl für ein positives familiäres Umfeld kontrolliert wurde (gute Beziehungsqualität, elterliches Engagement, usw.) blieben die Ergebnisse stabil. Zwei weitere Studien zum Bildungserfolg von Kindern in unterschiedlichen Familienstrukturen fanden ebenfalls ähnliche Nachteile für gemeinsame und Stiefkinder in komplexen Stieffamilien (Ginther und Pollak 2004; Gennetian 2005).

Eine Untersuchung mit einem groß angelegten Datensatz, dem U.S. National Longitudinal Survey of Youth, unterschied detailliert Jugendliche mit verheirateten und unverheirateten Eltern, Jugendliche, die als gemeinsame Kinder in komplexen Stieffamilien oder Kernfamilien leben, Jugendliche, die mit einem alleinerziehenden Vater/einer alleinerziehenden Mutter leben, Stiefkinder in verheirateten oder nichtehelichen Stieffamilien sowie Jugendliche, die in anderen Familienkonstellationen (bei Großeltern, anderen Verwandten, in Pflege- oder Adoptivfamilien) lebten, und verglich deren antisoziales Verhalten (Apel und Kaukinen 2008). Insgesamt berichteten Jugendliche aus ,nichtintakten“ Familien (Alleinerziehende, Stieffamilien und sonstige Familienformen) mehr Problemverhalten als Jugendliche aus ,,intakten" Familien (verheiratete und nichteheliche Kernfamilie, komplexe Stieffamilien). Allerdings wurden auch Unterschiede zwischen gemeinsamen Kindern der Partner in komplexen Stieffamilien und in verheirateten Kernfamilien sichtbar: Jugendliche in komplexen Stieffamilien wiesen häufiger antisoziales Verhalten auf. Zudem ließen sich auch unter Kontrolle von antisozialem Verhalten im Vorjahr Zusammenhänge des antisozialen Verhaltens zu einem geringen Haushaltseinkommen, fehlender elterlicher Unterstützung, schwachen schulischen Leistungen und antisozialen Freunden feststellen. Wenngleich einige Effekte der Familienstruktur durch diese letztgenannten Faktoren erklärt werden konnten, bestanden für Jugendliche, die in „nichtintakten“ Familien sowie in komplexen Stieffamilien lebten, weiterhin signifikante Unterschiede im Vergleich zu Jugendlichen aus verheirateten Kernfamilien, auch unter Kontrolle von soziodemografischen Merkmalen. Die Ergebnisse geben mit Hinblick auf die große Stichprobengröße (mit 510 Jugendlichen in komplexen Stieffamilien) einen klaren Hinweis darauf, dass nicht nur die Partnerschaftskonstellation der Eltern, sondern auch die Geschwisterkonstellation für das Wohlergehen Jugendlicher ausschlaggebend ist.

Befunde von Tillman (2008) stützen diese Interpretation. Ihre Untersuchung zeigt, dass neben der Familienform auch die Geschwisterkonstellation relevant für den Bildungserfolg ist. Je länger Jugendliche mit Stief- oder Halbgeschwistern zusammenlebten, desto niedriger war der Bildungserfolg (Tillman 2008). Demnach könnten insbesondere früh gegründete komplexe Stieffamilien mit gewissen Risiken für die Entwicklung der Kinder verbunden sein.

\subsection{Zur Bedeutung familialer Ressourcen für das Wohlbefinden von Kindern}

Ökonomische Schwierigkeiten, allen voran Einkommensknappheit aber auch eine subjektiv empfundene ökonomische Belastung, werden schon seit langem als ein 
zentraler Risikofaktor für das Wohlbefinden von Kindern und Jugendlichen gesehen (Walper 2009; Conger et al. 2010; Kohen und Guèvremont 2014). Gerade die schwierige sozioökonomische Lage von Alleinerziehenden im Vergleich zu Familien mit zwei Elternteilen ist ein gut dokumentierter Befund (Bundesministerium für Arbeit und Soziales 2017). Aber auch Stieffamilien kämpfen mit ökonomischen Schwierigkeiten. Eine ländervergleichende Studie kommt zu dem Ergebnis, dass Stieffamilien in Frankreich und Westdeutschland häufiger ökonomischen Druck innerhalb der Familie verspüren als Kernfamilien in diesen Ländern, während sich in der russischen Förderation und Ostdeutschland signifikante Unterschiede eher zwischen Alleinerziehenden und anderen Familienformen nachweisen lassen (Kreyenfeld und Martin 2011). Umgekehrt besteht auch ein Zusammenhang zwischen finanziellem Druck und einem erhöhten Risiko von Trennungen/Scheidungen (Cherlin 1978; Amato und Rogers 1997; Poortman 2005).

Abgesehen von den Rahmenbedingungen der Familienstruktur und der sozioökonomische Lage von Familien ist das kindliche Wohlbefinden auch eng mit den Beziehungsqualitäten innerhalb einer Familie verbunden und profitiert von einem harmonisch-kohäsiven Familienklima, Wärme und Unterstützung seitens der Eltern sowie einer positiven Beziehung zwischen den Eltern und nicht zuletzt einer konfliktarmen elterlichen Zusammenarbeit in der Erziehung, dem sogenannten Coparenting (Walper et al. 2017). Eine große Anzahl von Studien unterstreicht den Zusammenhang von emotional sicheren, positiven Beziehungen innerhalb einer Familie und kindlichem Wohlbefinden (z.B. Stormshak et al. 2000; Amato und Fowler 2002; McKee et al. 2007).

Im Vergleich verschiedener Familienformen zeigen sich vielfach Vorteile von Kernfamilien gegenüber Alleinerziehenden und Stieffamilien hinsichtlich der Qualität der Eltern-Kind-Beziehungen und des elterlichen Erziehungsverhaltens (Hetherington und Jodl 1994; Hofferth und Anderson 2003; Amato 2010). Dabei darf nicht außer Acht gelassen werden, dass Belastungen des Erziehungsverhaltens in Trennungsfamilien nicht per se der Familienstruktur zuzuschreiben sind, sondern vielfach der angespannten ökonomischen Lage und Problemen in der elterlichen Kooperation geschuldet sind. Unterschiede je nach Familienstruktur sind vielfach nicht mehr signifikant, sobald für sozioökonomische Faktoren kontrolliert wird (Bronstein et al. 1993, S. 274).

In Stieffamilien kann der Umgang mit unterschiedlichen Erziehungsvorstellungen und -praktiken des leiblichen und sozialen Elternteils eine Herausforderung für die Partner darstellen, nicht zuletzt, wenn der neue Partner „Novize“ in der Elternrolle ist. Angesichts fehlender sozialer Normen für die Ausgestaltung von sozialer Elternschaft sind die Partner auf ihre eigene Verständigung verwiesen (Cherlin 1978). Allerdings scheinen Fragen der Erziehung und der praktischen Gestaltung des Familienalltags im Vorfeld einer Stieffamiliengründung kein vordergründiges Thema zwischen den neuen Partnern zu sein. So zeigen verschiedene Studien, dass neue Partner häufig naiv und unvorbereitet die Rolle des Stiefelternteils antreten (Ganong und Coleman 1989; Robertson 2008; Cartwright 2010). Dennoch leisten Stiefelternteile oft einen wichtigen und wertvollen Beitrag in der Erziehung (Coleman et al. 2000). Befunde der breit angelegten Fragile Families and Child Well-being Study (USA) verweisen sogar auf Vorteile für Stiefkinder gerade in Bezug auf das 
Engagement des Stiefvaters, insbesondere wenn die Partner miteinander verheiratet sind im Vergleich zu verheirateten leiblichen Vätern (Berger und McLanahan 2015). Zudem zeigt sich, dass eine gute Beziehungsqualität zum leiblichen Elternteil im Haushalt ein Prädiktor für eine gute Stiefeltern-Stiefkind Beziehung ist, selbst wenn für eine komplexe Familienstruktur kontrolliert wurde (King et al. 2014).

Einen breiteren Blick auf die Beziehungsqualität innerhalb einer Familie, die über dyadische Beziehungen hinausgeht und die sozial-emotionale Beziehungsqualität eines ganzen Familiensystems aufgreift, bietet das Familienklima. Ein unterstützendes, positives Familienklima erweist sich als eine wichtige Ressource für das Wohlbefinden von Kindern und Jugendlichen (Stocker et al. 2007; Phillips 2012). Eine Studie von Phillips (2012) mit 278 Jugendlichen ging der Frage nach, ob die Familienstruktur (Kernfamilie, Alleinerziehende oder Stieffamilie) oder das Familienklima entscheidend für das Wohlbefinden Jugendlicher ist. Erwartungsgemäß stand ein gutes Familienklima (hohe Zufriedenheit und wenig negative Gefühle innerhalb der Familie) in einem deutlichen positiven Zusammenhang zum Wohlbefinden der Jugendlichen, während sich weder die untersuchten Aspekte von Wohlbefinden (z. B. Selbstwertgefühl, Optimismus, Hoffnungslosigkeit usw.) noch das Familienklima in den verschiedenen Familienstrukturen unterschieden. Dieses Ergebnis stützt auch eine Studie von Lansford et al. (2001), die ebenfalls zu dem Schluss kommen, dass innerfamiliale Prozesse der Beziehung und Interaktion, die in jeder Familie vorkommen, wichtiger für das Wohlbefinden der einzelnen Familienmitglieder sind als die Familienstruktur (Kernfamilie, Kernfamilie mit adoptierten Kindern, Stiefmutterfamilie, Stiefvaterfamilie und Alleinerziehende).

Umgekehrt ist ein hohes Konfliktniveau der Familie mit Belastungen des kindlichen Wohlbefindens verbunden. Konflikte zwischen den Eltern, die auch oft mit Problemen im Erziehungsverhalten und der Eltern-Kind-Beziehung einher gehen (Erel und Burman 1995), weisen einen vielfach dokumentierten Zusammenhang zu emotionalen Belastungen und Verhaltensproblemen der Kinder auf (z. B. Cummings und Davies 2002; Harold und Sellers 2018). Dies gilt auch für Stieffamilien (Broberg 2012). Studien über familiäre Konflikte, die nicht explizit auf die Eltern-Kindoder auf die Paardyade fokussieren, sondern Konflikte auf Ebene des Familienklimas untersuchen, sind jedoch selten. Eine Studie von Borrine et al. (1991) untersuchte die Verhaltensregulation Jugendlicher im Kontext verschiedener Familienkonstellation und familiärer Konflikte. Die Ergebnisse zeigten keine eigenständigen Effekte der Familienkonstellationen (Alleinerziehend, Stieffamilien und Kernfamilien), während dauerhafte familiäre Konflikte mit Beeinträchtigungen der Selbstregulierung Jugendlicher einhergingen. Auch die Interaktion zwischen Familienkonstellationen und familiärer Konflikte war statistisch unbedeutend, d.h., Konflikte kamen in allen Familienkonstellationen gleichermaßen zum Tragen.

Zusammenfassend legen diese Befunde nahe, dass Nachteile von Kindern aus Trennungsfamilien zu einem beträchtlichen Anteil auf Belastungen der ökonomischen Ressourcen und des Familienklimas zurückzuführen sind. Kinder in komplexen Stieffamilien könnten jedoch auch darüber hinaus mehr Nachteile erleben als Gleichaltrige in Kernfamilien. Bislang fehlen einschlägige Studien hierzu in Deutschland. Entsprechend sollen diese Fragen hier aufgegriffen werden. 


\subsection{Forschungsfragen und Hypothesen}

Während zahlreiche Studien nachteilige Effekte einer elterlichen Trennung für das Wohlbefinden von Kindern Alleinerziehender und in Stieffamilien aufzeigen, beleuchten nur wenige Studien die Situation von Kindern speziell in komplexen Stieffamilien. Die hierzu verfügbaren Befunde legen nahe, dass nicht nur Stiefkinder, sondern auch gemeinsame Kinder in komplexen Stieffamilien erhöhte Belastungen ihres Wohlbefindens aufweisen. Bislang ließen sich diese Nachteile nicht durch erhöhte Belastungen der Eltern-Kind-Beziehung und des Erziehungsverhaltens aufklären. Ob mögliche Beeinträchtigungen des weiter gefassten Familienklimas besseren Aufschluss bieten, soll hier geprüft werden.

Die vorliegende Studie untersucht das kindliche Wohlbefinden in verschiedenen Familienformen und vergleicht einfache Stiefvaterfamilien, komplexe Stieffamilien, Familien mit einer alleinerziehenden Mutter und Kernfamilien. Zudem berücksichtigen wir die ökonomische Lage und die Beziehungsqualität innerhalb einer Familie. Daraus ergeben sich folgende, aus der Literatur abgeleitete, Hypothesen:

1. Kinder in Kernfamilien berichten über ein höheres Wohlbefinden als Kinder in Trennungsfamilien und insbesondere jene in komplexen Stieffamilien.

2. Kindliches Wohlbefinden hängt positiv mit einem emotional warmen und kindzentrierten elterlichen Erziehungsverhalten, einem harmonischen Familienklima und wenig Konflikten innerhalb der Familie zusammen.

3. Ökonomische Probleme der Familie gehen mit einem geringeren kindlichen Wohlbefinden einher.

4. Der Zusammenhang zwischen Familienform und kindlichem Wohlbefinden wird durch die finanzielle Situation der Familie und die Qualität der Familienbeziehungen vermittelt (Mediatorhypothese). Während eine schwierige ökonomische Situation vor allem die Nachteile von Kindern in alleinerziehenden Familien erklären sollte, ist zu erwarten, dass Belastungen des Familienklimas als bedeutsamer Mediator von geringerem Wohlbefinden für Kinder in komplexen Stieffamilien fungieren.

\section{Methodisches Vorgehen}

\subsection{Datenbasis und Stichprobe}

Zur Beantwortung der Forschungsfrage wird die erste Welle des Surveys „Aufwachsen in Deutschland: Alltagswelten“ (AID:A 2009) verwendet (Rauschenbach und Bien 2012). Der AID:A-Survey ist eine für Deutschland repräsentative Studie mit telefonischer Befragung von über 25.000 (Ziel)Personen im Alter ab Geburt bis 55 Jahren. Kinder ab einem Alter von neun Jahren wurden persönlich über relevante Themen ihres Alltags befragt. Soziodemografische und strukturelle Daten wurden für Minderjährige von einem Elternteil - meist der Mutter - erhoben.

Für die Stichprobe wurden 2173 Zielkinder zwischen neun und zwölf Jahren ausgewählt, die an der Kinderbefragung des AID:A-Surveys teilnahmen. 21 Kinder 
wurden aus dieser Stichprobe ausgeschlossen, da sie keiner Familienform eindeutig zugeordnet werden konnten. Zudem ist die Anzahl der Kinder, die ausschließlich beim leiblichen Vater oder in einer Stiefmutterfamilie leben sehr gering $(n=8)$; sie wurden daher nicht einbezogen. Für die folgenden Analysen ergibt sich somit eine Stichprobe von 2144 Zielpersonen, darunter 51,1\% Jungen und 48,9\% Mädchen. Das Durchschnittsalter der befragten Kinder lag bei 10,5 Jahren ( $\mathrm{SD}=1,11)$.

Erwartungsgemäß lebt die überwiegende Mehrheit der Kinder in Kernfamilien $(81,3 \%, n=1744)$. Die zweitgrößte Gruppe bilden Kinder, die mit einer alleinerziehenden Mutter zusammenleben $(11,9 \%, n=255)$. Schließlich leben 6,8\% $(n=145)$ aller Kinder in einer Stiefvaterfamilie mit einer biologischen Mutter und ihrem neuen Partner (Stiefvater). In dieser letztgenannten Gruppe können mit Hilfe des AID:A-Survey auch komplexe Stieffamilien identifiziert werden, nämlich solche, in denen Kinder aus früheren Beziehungen von einem Partnern leben und zusätzlich gemeinsame Kinder der neuen Partner in diese Stieffamilienkonstellation geboren wurden. Dies trifft auf 58,6\% $(n=85)$ aller Kinder zu, die in einer Stiefvaterfamilie leben. Aufgrund dieser geringen Fallzahl von Kindern in komplexen Stieffamilien war es nicht möglich, diese weiter nach gemeinsamen $(n=58)$ oder Stiefkindern $(n=27)$ zu differenzieren. Für die nachstehende Analyse ergeben sich somit vier Familienformen, in denen die ausgewählten Zielkinder leben: (1) Kernfamilienkinder (Referenzkategorie), (2) Kinder, die mit einer alleinerziehenden Mutter leben, (3) Stiefkinder in einer einfachen Stiefvaterfamilie (ohne zusätzliche gemeinsame Kinder) und (4) Kinder, die in komplexen Stieffamilien leben (entweder als Stiefkind oder als gemeinsames Kind beider Partner).

\subsection{Indikatoren und Auswertung}

Für die vorliegende Studie wurde als Indikator für kindliches Wohlbefinden der „Strenghts and Difficulties Questionaire“ (SDQ) verwendet (Goodman 1997). Der SDQ ist ein Instrument zur Erfassung der psychischen Gesundheit (vs. Auffälligkeiten) bei Kindern und Jugendlichen im Alter von vier bis 16 Jahren. Neben einer Eltern- und Lehrerversion existiert auch ein Fragebogen, den Kinder und Jugendliche selbst beantworten und der hier zum Einsatz kommt. Dieses Instrument umfasst vier problemorientierte Subskalen zu je fünf Items: emotionale Probleme (z. B. ,Ich habe häufig Kopfschmerzen oder Bauchschmerzen; mir wird oft schlecht"), Verhaltensprobleme (z. B. „Ich werde leicht wütend; ich verliere oft meine Beherrschung“"), Hyperaktivität (z. B. ,Ich bin oft unruhig; ich kann nicht lange stillsitzen“") und Verhaltensprobleme mit Gleichaltrigen (z. B. „Ich bin meistens für mich alleine; ich beschäftige mich lieber mit mir selbst") sowie eine Subskala, die prosoziales Verhalten abdeckt mit ebenfalls fünf Items (z. B. „Ich versuche, nett zu anderen Menschen zu sein, ihre Gefühle sind mir wichtig“). Für die Berechnung des Gesamtproblemwertes wurden die vier problemorientierten Subskalen zusammengefasst (Cronbachs Alpha: 0,78), prosoziales Verhalten wurde nicht berücksichtigt. Die Cut-off Werte sind analog zu Goodman (1997) gewählt (0-13 Punkte: unauffällig; 14-16 Punkte: grenzwertig; 17-40 Punkte: auffällig). Werte im Bereich ,unauffällig“ werden hier als Indikator für kindliches Wohlbefinden verwendet, während grenzwertiges oder auffälliges Verhalten als Belastung des kindlichen Wohlbefindens eingestuft 
wird $(1=$ kindliches Wohlbefinden, $0=$ kindliche Belastung). In der hier verwendeten Stichprobe berichten 83,2\% der Kinder Werte im Bereich des Wohlbefindens, während 16,8\% der Gruppe der auffälligen/grenzwertigen Kinder zuzuordnen sind. Dies entspricht weitgehend der Verteilung bei Goodmann (1997), der $80 \%$ der Kinder als ,unauffällig“ einstufte.

Dem Indikator Armutsrisiko liegt das bedarfsgewichtete Äquivalenzeinkommen der Personen im Haushalt zu Grunde. Hierbei sind die Anzahl und das Alter der Personen im Haushalt als Bedarfsmerkmale sowie Einspareffekte durch gemeinsames Wirtschaften in Rechnung gestellt. Der Schwellenwert für Armutsgefährdung liegt nach EU-Definition bei $60 \%$ des Medianwerts des bedarfsgerechten Äquivalenzeinkommens in der Bevölkerung. Er teilt die Stichprobe in zwei Gruppen $(0=$ oberhalb der Armutsschwelle und 1= unterhalb der Armutsschwelle).

Die Beziehungsqualitäten innerhalb einer Familie wurden im AID:A-Survey durch die Kinder erfasst. Ein Indikator für die Beziehungsqualität ist die Beziehung zwischen Kind und leiblicher Mutter, die durch eine Kurzversion der Supportive Parenting Scale (Simons et al. 1992) durch sechs Items erfasst wurde (z. B. ,Wie oft spricht Deine Mutter mit dir über das, was du tust oder erlebt hast?", Antwortrating von $1=$ nie bis $4=$ immer). Die interne Konsistenz des Instruments ist akzeptabel (Cronbach's Alpha 0,68) und entspricht weitgehend den Werten der Originalskala (mit 9 Items). Das Familienklima wurde angelehnt an Moos (1974) erfasst, wobei drei Items zur Kohäsion (z. B. „Ich bin gerne mit meiner Familie zusammen“, Cronbach's Alpha 0,61) und ein Item zu Konflikten in der Familie (,In unserer Familie kommt es zu Reibereien.") einbezogen wurden (mit ebenfalls vierstufigem Rating von $1=$ nie bis $4=$ immer). Die hier berichteten Konflikte beziehen sich auf eine Gesamteinschätzung der Konflikte innerhalb der Familie.

Als Kontrollvariablen werden die Bildung der Mutter bzw. des Auskunft gebenden Elternteils (nur ca. 3\% der Kernfamilien war dies der Vater), das Alter des Kindes (in Jahren) und das Geschlecht des Kindes (1= männlich, 2= weiblich) in die Analyse einbezogen. Die elterliche Bildung wurde mit Hilfe der Comparative Analysis of Social Mobility in Industrial Nations (CASMIN) klassifiziert (König et al. 1987). Für die Analysen werden einzelne Kategorien aufgrund geringer Fallzahlen zusammengefasst (in Klammer jeweils die Verteilung für das Analysesample): $1=$ kein Abschluss/Hauptschulabschluss sowie sonstige Abschlüsse mit und ohne Berufsausbildung (15,1\%), 2= Mittlere Reife mit und ohne Berufsausbildung (36,7\%), $3=$ Abitur mit und ohne Berufsausbildung (20,3\%) und $4=$ Fachhochschulabschluss und Universitätsabschluss $(27,8 \%)$.

Für die Beantwortung der Forschungsfragen wurden einerseits deskriptive Verfahren wie Chi-Quadrat-Tests, Korrelationen und ANOVAs und andererseits multifaktorielle Verfahren wie lineare und logistische Regressionen eingesetzt. Im multivariaten Modell werden die Zusammenhänge mit kindlichem Wohlbefinden mit Hilfe von logistischen Regressionen geprüft, wobei die Prädiktoren im Modellvergleich schrittweise aufgenommen werden, um mögliche indirekte Zusammenhänge aufzeigen zu können. Um die Signifikanz von indirekten Effekten überprüfen zu können, wurde die Sobel Teststatistik verwendet (Baron und Kenny 1986). 


\section{Ergebnisse}

\subsection{Deskriptive Ergebnisse zum Vergleich der Familienformen und Zusammenhänge zum Wohlbefinden der Kinder}

Wie zu erwarten, zeigen sich signifikante Gruppenunterschiede zwischen den einzelnen Familienformen, vor allem in der Anzahl der Kinder im Haushalt und dem Armutsrisiko des Haushalts, aber auch in weiteren Merkmalen von Eltern und Kindern (siehe Tab. 1). In komplexen Stieffamilien fällt die Kinderzahl am höchsten und in Familien mit alleinerziehender Mutter am geringsten aus. Für Kinder, die bei einer alleinerziehenden Mutter leben, ist das Armutsrisiko am höchsten, dicht gefolgt von Kindern in komplexen Stieffamilien, während Kinder in Kernfamilien und - entgegen unseren Erwartungen - vor allem jene in einfachen Stiefvaterfamilien deutlich seltener von Armut betroffen sind. Generell ist das Bildungsniveau der Auskunft gebenden Eltern (i.d.R. der Mütter) vergleichsweise hoch, aber Mütter in komplexen Stieffamilien berichten einen geringeren Anteil an Fachhochschul- oder

Tab. 1 Deskriptive Zusammenhänge der Familienform

\begin{tabular}{|c|c|c|c|c|}
\hline & $\begin{array}{l}\text { Kernfami- } \\
\text { lienkinder }\end{array}$ & $\begin{array}{l}\text { Kinder von al- } \\
\text { leinerziehenden } \\
\text { Müttern }\end{array}$ & $\begin{array}{l}\text { Stiefkinder aus } \\
\text { Stiefvaterfami- } \\
\text { lien }\end{array}$ & $\begin{array}{l}\text { Kinder aus kom- } \\
\text { plexen Stiefvater- } \\
\text { familien }\end{array}$ \\
\hline $\begin{array}{l}\text { Anzahl der Kinder } \\
\text { im Haushalt }(\mathrm{F}(3 \text {, } \\
2144)=30,98 ; p<0,001)\end{array}$ & $\begin{array}{l}M=2,24 \\
(S D=0,89)\end{array}$ & $\begin{array}{l}M=1,87 \\
(S D=0,93)\end{array}$ & $\begin{array}{l}M=1,81 \\
(S D=0,77)\end{array}$ & $\begin{array}{l}M=2,73 \\
(S D=0,90)\end{array}$ \\
\hline $\begin{array}{l}\text { Armutsrisiko des Haus- } \\
\text { halts }\left(\chi^{2}=46,20 ; \mathrm{df}=3 ;\right. \\
p<0,001 ; N=2009)\end{array}$ & $13,7 \%$ & $28,8 \%$ & $5,2 \%$ & $24,4 \%$ \\
\hline \multicolumn{5}{|c|}{ Bildung der Mutter (i.d. R. als antwortender Elternteil) $\left(\chi^{2}=22,09 ; \mathrm{df}=9 ; p<0,001, N=2144\right)$} \\
\hline $\begin{array}{l}\text { Kein Abschluss/ } \\
\text { Hauptschulabschluss so- } \\
\text { wie sonstige Abschlüsse }\end{array}$ & $14,6 \%$ & $18,4 \%$ & $10,0 \%$ & $20,0 \%$ \\
\hline Mittlere Reife ${ }^{\mathrm{a}}$ & $36,6 \%$ & $31,4 \%$ & $41,7 \%$ & $50,6 \%$ \\
\hline Abitur $^{\mathrm{a}}$ & $19,9 \%$ & $24,7 \%$ & $21,7 \%$ & $15,3 \%$ \\
\hline $\begin{array}{l}\text { Fachhochschul- und Uni- } \\
\text { versitätsabschluss }\end{array}$ & $28,9 \%$ & $25,5 \%$ & $26,7 \%$ & $14,1 \%$ \\
\hline Gesamt & $100 \%$ & $100 \%$ & $100 \%$ & $100 \%$ \\
\hline $\begin{array}{l}\text { Kindliches Wohlbefin- } \\
\text { den }\left(\chi^{2}=42,34 ; \mathrm{df}=3 ;\right. \\
p<0,001 ; N=2142)\end{array}$ & $85,4 \%$ & $76,1 \%$ & $80,0 \%$ & $62,4 \%$ \\
\hline $\begin{array}{l}\text { Mutter-Kind-Beziehung } \\
(\mathrm{F}(3,2144)=2,26 \\
p>0,05)\end{array}$ & $\begin{array}{l}M=3,43 \\
(\mathrm{SD}=0,41)\end{array}$ & $\begin{array}{l}M=3,40 \\
(S D=0,42)\end{array}$ & $\begin{array}{l}M=3,43 \\
(S D=0,40)\end{array}$ & $\begin{array}{l}M=3,33 \\
(S D=0,44)\end{array}$ \\
\hline $\begin{array}{l}\text { Familienklima }(\mathrm{F}(3, \\
2144)=9,03 ; p<0,001)\end{array}$ & $\begin{array}{l}M=3,55 \\
(\mathrm{SD}=0,44)\end{array}$ & $\begin{array}{l}M=3,42 \\
(\mathrm{SD}=0,53)\end{array}$ & $\begin{array}{l}M=3,64 \\
(\mathrm{SD}=0,50)\end{array}$ & $\begin{array}{l}M=3,42 \\
(S D=0,39)\end{array}$ \\
\hline $\begin{array}{l}\text { Streit in der Familie }(\mathrm{F}(3, \\
2086)=6,90 ; p<0,001)\end{array}$ & $\begin{array}{l}M=1,96 \\
(\mathrm{SD}=0,60)\end{array}$ & $\begin{array}{l}M=2,08 \\
(\mathrm{SD}=0,54)\end{array}$ & $\begin{array}{l}M=1,97 \\
(S D=0,67)\end{array}$ & $\begin{array}{l}M=2,20 \\
(\mathrm{SD}=0,69)\end{array}$ \\
\hline
\end{tabular}

${ }^{a}$ Mit und ohne Berufsausbildung 
Universitätsabschlüssen sowie einen höheren Anteil an mittlerer Reife als Mütter in den drei Vergleichsgruppen.

Im Einklang mit Hypothese 1 fällt das kindliche Wohlbefinden in Kernfamilien am höchsten aus, d.h., diese Kinder berichten am häufigsten eine unbelastete seelische Gesundheit, gefolgt von Kindern in einfachen Stiefvaterfamilien und Kindern, die mit einer alleinerziehenden Mutter leben, während Kinder aus komplexen Stieffamilien am seltensten ein entsprechendes Wohlbefinden angeben. Die MutterKind-Beziehung unterscheidet sich hingegen nicht signifikant zwischen den Familienformen. Über alle Konstellationen hinweg schätzen die Kinder die Beziehung zur Mutter als sehr warm und kindzentriert ein. Wohl aber zeigen sich signifikante Unterschiede hinsichtlich der Kohäsion im Familienklima und in Bezug auf Streit in der Familie. Allerdings zeigen sich signifikante Unterschiede hinsichtlich des Familienklimas und in Bezug auf Streit in der Familie. Kinder, die mit einer alleinerziehenden Mutter oder in einer komplexen Stieffamilie leben, schätzen ihr Familienklima am niedrigsten ein, gefolgt von Kernfamilienkindern und Kindern, die in einer Stiefvaterfamilie leben. Gleichzeitig berichten Kinder in komplexen Stieffamilien aber auch die meisten Reibereien in der Familie, gefolgt von Kindern alleinerziehender Mütter, während Kernfamilien und einfache Stiefvaterfamilien nach Auskunft der Kinder die geringste Konfliktneigung aufwiesen.

In korrelativen Analysen zeigen sich signifikante aber zumeist schwache negative Zusammenhänge des kindlichen Wohlbefindens mit der Familienform (Alleinerziehende $r=-0,07 * *$; komplexe Stieffamilien $\left.r=-0,11^{* * *}\right)$, dem Armutsrisiko $\left(\mathrm{r}=-0,08^{* *}\right)$ sowie Streit in der Familie $(\mathrm{r}=-0,22 * * *)$ und positive Zusammenhänge mit dem Alter des Kindes $(\mathrm{r}=0,07 * *)$, der Bildung der Mutter $\left(\mathrm{r}=0,10^{* * *}\right)$, einer positiven Mutter-Kind-Beziehung $(\mathrm{r}=0,20 * * *)$ und einem kohäsiven Familienklima $\left(\mathrm{r}=0,22^{* * *}\right)$. Das Geschlecht, die Anzahl der Kinder und die Familienform in Form einer einfachen Stiefvaterfamilie hängen hingegen nicht signifikant mit kindlichem Wohlbefinden zusammen. Das höhere Wohlbefinden der Kinder bei positiven Familienbeziehungen und bei Abwesenheit von Armut entspricht Hypothese 2 und 3.

\subsection{Multifaktorielle Ergebnisse}

Welches relative Gewicht haben nun diese Faktoren für das kindliche Wohlbefinden? Mit Hilfe von logistischen Regressionen wird durch schrittweise Hinzunahme von Prädiktoren kindliches Wohlbefinden (SDQ, s. oben) vorhergesagt. Tab. 2 zeigt drei Modelle, wobei das erste Modell lediglich die Kontrollvariablen sowie die Familienform einbezieht, Modell 2 zusätzlich Armut berücksichtigt und Modell 3 auch die Indikatoren der Familienbeziehungen aufnimmt. Mit Blick auf die soziodemografischen Kontrollvariablen erweisen sich lediglich zwei Faktoren als bedeutsam. So fällt das kindliche Wohlbefinden mit zunehmenden Alter und höherer mütterlicher Bildung besser aus, allerdings besteht kein Zusammenhang zwischen dem Geschlecht und der Anzahl der Kinder im Haushalt und kindlichem Wohlbefinden. Diese Zusammenhänge ändern sich kaum über die drei Modelle hinweg.

Wie in Tab. 2 ersichtlich, ist die Familienform ein zentraler Faktor für kindliches Wohlbefinden, selbst wenn für soziodemografische Merkmale (Modell 1), Armutsrisiko (Modell 2) und die Qualität familialer Beziehungen (Modell 3) kontrolliert 
Tab. 2 Zusammenhänge mit kindlichem Wohlbefinden - Ergebnisse logistischer Regressionen

\begin{tabular}{|c|c|c|c|}
\hline & Model 1 & Model 2 & Model 3 \\
\hline Alter des Kindes in Jahren & $1,18 * *$ & $1,18 * *$ & $1,19 * * *$ \\
\hline Geschlecht des Kindes & 1,15 & 1,14 & 1,12 \\
\hline Anzahl der Kinder im Haushalt & 0,90 & 0,92 & 1,04 \\
\hline $\begin{array}{l}\text { Bildung der Mutter (i.d. R. als antworten- } \\
\text { der Elternteil) }\end{array}$ & - & - & - \\
\hline Ref.: FH- oder Universitätsabschluss & - & - & - \\
\hline Kein Abschluss/HS-Abschluss & $0,42 * * *$ & $0,45 * * *$ & $0,41 * * *$ \\
\hline Mittlere Reife & $0,59 * *$ & $0,61 * *$ & $0,55^{* *}$ \\
\hline Abitur & $0,59 * *$ & $0,60 * *$ & $0,58 * *$ \\
\hline Familienform & - & - & - \\
\hline Ref.: Kernfamilie & - & - & - \\
\hline Alleinerziehende Mutter & $0,55^{* *}$ & $0,58 * *$ & 0,72 \\
\hline Stiefvaterfamilie & 0,59 & 0,58 & 0,56 \\
\hline Komplexe Stieffamilie & $0,33^{* * * *}$ & $0,33^{* * *} *$ & $0,41 * *$ \\
\hline Armutsrisiko & - & 0,78 & 0,76 \\
\hline Familienklima & - & - & $2,17 * * *$ \\
\hline Streit in der Familie & - & - & $0,46 * * *$ \\
\hline Mutter-Kind-Beziehung & - & - & $1,84 * *$ \\
\hline Nagelkerke's $\mathrm{R}^{2}$ & 0,055 & 0,056 & 0,180 \\
\hline
\end{tabular}

$N=1954, * p<0,05 * * p<0,01 * * * p<0,001 ; 1=$ kindliches Wohlbefinden, $0=$ kindliche Belastung

wird. In Einklang mit Hypothese 1 berichten Kinder in komplexen Stieffamilien über alle Modelle hinweg seltener ein positives Wohlbefinden als Kinder in Kernfamilien. Dieser Effekt schwächt sich zwar von einem Odds-Ratio-Wert (OR) von 0,33 auf $0,41 \mathrm{ab}$, bleibt aber auch in Modell 3 bestehen. Auch Kinder mit alleinerziehender Mutter berichten seltener ein unbelastetes Wohlbefinden (Modell 1), selbst unter Kontrolle des Armutsrisikos (Modell 2). Werden die Indikatoren Familienklima, Streit in der Familie und Mutter-Kind-Beziehung in das Modell aufgenommen (Modell 3), so nivellierten sich die Unterschiede zwischen Ein-Eltern- und Kernfamilien vollständig. Wie laut Hypothese 2 erwartet, steigt die Chance für ein höheres kindliches Wohlbefinden, wenn die Kinder ein gutes Familienklima, wenig familiale Konflikte und eine gute Beziehungsqualität zur Mutter berichten, wobei alle drei Indikatoren der Familienbeziehungen trotz ihrer Interdependenzen einen eigenständigen Zusammenhang zum Wohlbefinden der Kinder aufweisen.

Diese Ergebnisse legen nahe, dass der Zusammenhang von Familienform und kindlichem Wohlbefinden über die Qualität der Familienbeziehungen vermittelt wird (Mediatorhypothese). Dies gilt im Besonderen für Kinder mit alleinerziehender Mutter (vollständige Mediation) aber auch für Kinder in einer komplexen Stieffamilie (partielle Mediation). Die Prüfung der Mediatoreffekte bestätigt signifikante indirekte Zusammenhang des Familientypes mit kindlichem Wohlbefinden, die über das Familienklima (Sobel-Teststatistik: Alleinerziehende: $-3,92 * * *(\mathrm{SD}=0,01)$; komplexe Stieffamilie: $-2,30 *(\mathrm{SD}=0,01))$ und Streit in der Familie (Sobel Teststatistik: Alleinerziehende: $-2,55 *(\mathrm{SD}=0,01)$; komplexe Stieffamilie: $-3,26 * *(\mathrm{SD}=0,01))$ 
vermittelt werden. Demgegenüber weist die Beziehungsqualität zur Mutter nur in komplexen Stieffamilien (Sobel Teststatistik: $-2,20 *(\mathrm{SD}=0,01)$ ), einen signifikanten indirekten Effekt auf.

Obwohl das Armutsrisiko der Familie bivariat signifikant negativ mit dem kindlichen Wohlbefinden zusammenhängt (s. oben), bleibt dieser Effekt bei der Hinzunahme von weitern Kontrollvariablen nicht bestehen (siehe Tab. 2, Modell 2). Darüber hinaus wurde getestet, ob Armut in Interaktion mit der Familienform einen bedeutsamen Effekt auf kindliches Wohlbefinden hat. Der Interaktionseffekt von Armut und Familientyp verbessert jedoch weder die Modellgüte (Nagelkerkes $\mathrm{R}^{2}$ ) noch erweist er sich als signifikanter Faktor für kindliches Wohlbefinden. Auch erbrachten lineare Regressionen keine Zusammenhänge zwischen dem Armutsrisiko und den Indikatoren der Beziehungsqualität. Insofern weist das Armutsrisiko hier weder einen direkten oder indirekten Zusammenhang mit kindlichem Wohlbefinden auf, noch vermittelt es den Zusammenhang zwischen der Familienstruktur und dem Wohlbefinden der Kinder. Insofern muss Hypothese 3 vollständig und Hypothese 4 nur bezüglich des Armutsrisikos abgelehnt werden.

Insgesamt verbessert sich in den schrittweisen Regressionen der Kennwert für die Modellgüte (Nagelkerkes $\mathrm{R}^{2}$ ) durch die Hinzunahme von weiteren Prädiktoren im Vergleich von Modell 1 und 2 einerseits und Modell 3 andererseits und kann in Modell 3 als zufriedenstellend interpretiert werden.

\section{Diskussion}

Die Studie hatte das Ziel, die Bedeutung der Familienstruktur für das kindliche Wohlbefinden zu beleuchten und hierbei speziell die Situation komplexer Stieffamilien in den Blick zu nehmen. Während Stieffamilien neben Alleinerziehenden und Kernfamilien als eine vermeintlich einheitliche Gruppe ausgewiesen werden, konnten wir mit den Daten des AID:A-Surveys (2009) den Fokus auf Binnendifferenzierungen innerhalb der Stieffamilien richten und neben Kernfamilien und alleinerziehenden Müttern sowohl einfache Stiefvaterfamilien als auch komplexe Stieffamilien mit Stief- und/oder Halbgeschwistern vergleichen. Zudem wurde geprüft, inwieweit sich mögliche Unterschiede im Wohlbefinden der Kinder aus verschiedenen Familienstrukturen auf entsprechende Unterschiede im Armutsrisiko und in den Familienbeziehungen zurückführen lassen. Um der eigenständigen Perspektive von Kindern Rechnung zu tragen, wurden deren Angaben zum Wohlbefinden sowie zur Qualität der Familienbeziehungen in den Mittelpunkt gestellt (Walper et al. 2017). Damit liefert die Studie auch im Feld internationaler Familienforschung einen wichtigen Beitrag zur differenzierten Betrachtung von Nachtrennungsfamilien.

Im Einklang mit den wenigen verfügbaren internationalen Studien (Gennetian 2005; Apel und Kaukinen 2008; Halpern-Meekin und Tach 2008; Brown et al. 2015) lassen auch die hier berichteten Befunde darauf schließen, dass Kinder in komplexen Stieffamilien hinsichtlich ihres Wohlbefindens die stärksten Nachteile gegenüber Kindern in Kernfamilien aufweisen. Dies gilt selbst unter Kontrolle sozioökonomischer Ressourcen wie der Bildung der Mutter, die in komplexen Stieffamilien deutlich geringer ausfällt als in anderen Familienformen. Während sich die 
elterliche Bildung insgesamt als eine zentrale Ressource für das Wohlbefinden von Kindern erweist, hat das Armutsrisiko keinen eigenständigen Erklärungsbeitrag. Erwartungsgemäß fungieren ein weniger harmonisches Familienklima und vermehrte Konflikte als partielle Mediatoren, unter deren Kontrolle sich die Nachteile im Wohlbefinden von Kindern in komplexen Stieffamilien vermindern. Allerdings können diese Nachteile durch die hier berücksichtigen Mediatoren nicht gänzlich aufgeklärt werden.

Auch Kinder alleinerziehender Mütter berichten häufiger von Beeinträchtigungen ihres Wohlbefindens als Kinder in Kernfamilien. Anders als bei Gleichaltrigen aus komplexen Stieffamilien lässt sich das geringe Wohlbefinden von Kindern Alleinerziehender vollständig durch die höheren Belastungen der Familienbeziehungen einem schlechteren Familienklima und Streit in der Familie - erklären. Dies entspricht der Befundlage vieler Studien, nach denen innerfamilialen Prozesse hohe Relevanz für die Entwicklung von Kindern und Jugendlichen zukommt, während sich die Familienform darüber hinaus als wenig bedeutender eigenständiger Faktor erweist (Borrine et al. 1991; Phillips 2012). Eine differenzierte Betrachtung der Familienformen fehlt allerdings zumeist. Unsere Ergebnisse legen nahe, dass gängige Indikatoren des Familienklimas und der Eltern-Kind-Beziehung zwar die vermehrten Belastungen Alleinerziehender abbilden, die für das Wohlbefinden der Kinder relevant sind, dass jedoch die spezifischen Herausforderungen des Zusammenlebens in komplexen Stieffamilien nicht vollständig durch diese Aspekte der Familienbeziehungen erklärt werden.

Die Gründung einer Stieffamilie birgt ohnehin Asymmetrien der Eltern-KindVerhältnisse, da die Hauptverantwortung für die Kinder überwiegend - zumindest in Stiefvaterfamilien - bei dem leiblichen Elternteil (der Mutter) verbleibt. Vermutlich verschärften sich diese Asymmetrien, wenn Geschwister unterschiedliche Kindschaftsverhältnisse zu beiden Partnern aufweisen, da Kinder nun unterschiedliche „Zuständigkeiten“ und vermutlich auch Zuwendungen der Eltern erfahren. Hinzu kommen unterschiedliche Kontakte und Einbindungen der Geschwister in „externe Familien“ getrennt lebender Elternteile. Wenngleich ein guter innerfamilialer Zusammenhalt in der Stieffamilie und eine wertschätzende Eltern-Kind-Beziehung helfen, mit dieser Situation besser umzugehen, ist es durchaus wahrscheinlich, dass darüber hinaus weitere Aspekte der Lebenssituation komplexer Familienformen berücksichtigt werden müssen. Subtile soziale Vergleiche unter Geschwistern, Ungleichbehandlungen, die zu erlebten Ungerechtigkeiten werden können, aber auch das Gefühl der gemeinsamen Kinder, die Familie zusammenzuhalten zu müssen, können durchaus mit vermehrten Belastungen der Kinder einhergehen.

Überraschenderweise zeigt das Armutsrisiko in der multivariaten Zusammenschau keinen eigenständigen Zusammenhang zum Wohlbefinden der Kinder. Vor dem Hintergrund vielfältiger anderslautender Befunde der Armutsforschung zu Kindern ist dies erklärungsbedürftig. Dies gilt umso mehr, als der Zusammenhang nicht nur dann fehlt, wenn zugleich auch die Familienbeziehungen berücksichtigt werden, was im Sinne des Familien-Stress-Modells (Conger et al. 2010) auf einen indirekten Effekt von Armut über Belastungen der Familienbeziehungen verweisen könnte. Er fehlt auch dann, wenn die Bedeutung des Armutsrisikos nur im Kontext demographischer Faktoren betrachtet wird. Möglicherweise reflektieren die Abstufungen im 
elterlichen Bildungsniveau in stärkerem Maße relevante Unterschiede in der Ressourcenausstattung der Familie als der einfache Fokus auf das Armutsrisiko. Auch ist nicht auszuschließen, dass die verfügbaren Einkommensdaten nur unzureichenden Einblick in die finanzielle Situation der Familien geben. Weitergehende Analysen mit der zweiten Welle des AID:A Surveys legen die Vermutung nahe, dass der erlebte finanzielle Druck im Haushalt für Kinder weitaus bedeutender ist als das Armutsrisiko, das Konsumbedarfe lediglich pauschaliert dem Haushaltseinkommen gegenüber stellt und erlebte Engpässe nicht direkt einfängt (Entleitner-Phleps und Walper 2019).

Zuletzt sind Limitationen dieser Untersuchung zu erwähnen. Die Stichprobe des AID:A-Surveys weist einen merklichen Bildungsbias auf, d.h., Befragte mit höherer Bildung sind überrepräsentiert. Effekte geringer sozioökonomischer Ressourcen könnten insofern unterschätzt werden. Darüber hinaus erlauben unsere Querschnittdaten keine Interpretationen hinsichtlich der Kausalität der Zusammenhänge. Kritisch anzumerken ist auch, dass lediglich Kurzindikatoren mit nur zufriedenstellender Reliabilität einzelner Skalen einbezogen werden konnten - ein gängiger Nachteil der Surveyforschung, der durch große Stichprobenumfänge nur bedingt wettgemacht wird. Schließlich haben wir kindliches Wohlbefinden ausschließlich an der seelischen Gesundheit - gemessen mit dem international etablierten Instrument des Strenghts and Difficulties Questionaire (SDQ) - festgemacht. Weitere Aspekte wie die Zufriedenheit der Kinder mit unterschiedlichen Lebensbereichen, ihr Selbstwertgefühl und Kompetenzerleben wären wichtige Ergänzungen. Trotz dieser Einschränkungen liefern die Ergebnisse dieser Studie wichtige Erkenntnisse über das Wohlbefinden von Kindern in unterschiedlichen Familienkonstellationen und darüber, welche weiteren Faktoren diesbezügliche Unterschiede zwischen Kindern aus unterschiedlichen Familienformen erklären könnten. Wir hoffen, mit unseren Analysen den Anstoß zu weiterführenden Arbeiten zu geben, nicht zuletzt zu Panelanalysen, die es erlauben die Familienentwicklung und Verhandlung der Elternrollen in Stieffamilien im Zeitverlauf zu beobachten, um noch aussagekräftigere Erkenntnisse zu gewinnen.

Funding Open Access funding provided by Projekt DEAL.

Open Access Dieser Artikel wird unter der Creative Commons Namensnennung 4.0 International Lizenz veröffentlicht, welche die Nutzung, Vervielfältigung, Bearbeitung, Verbreitung und Wiedergabe in jeglichem Medium und Format erlaubt, sofern Sie den/die ursprünglichen Autor(en) und die Quelle ordnungsgemäß nennen, einen Link zur Creative Commons Lizenz beifügen und angeben, ob Änderungen vorgenommen wurden.

Die in diesem Artikel enthaltenen Bilder und sonstiges Drittmaterial unterliegen ebenfalls der genannten Creative Commons Lizenz, sofern sich aus der Abbildungslegende nichts anderes ergibt. Sofern das betreffende Material nicht unter der genannten Creative Commons Lizenz steht und die betreffende Handlung nicht nach gesetzlichen Vorschriften erlaubt ist, ist für die oben aufgeführten Weiterverwendungen des Materials die Einwilligung des jeweiligen Rechteinhabers einzuholen.

Weitere Details zur Lizenz entnehmen Sie bitte der Lizenzinformation auf http://creativecommons.org/ licenses/by/4.0/deed.de. 


\section{Literatur}

Albert, M., Hurrelmann, K., \& Quenzel, G. (2019). Shell-Jugendstudie: Jugend 2019. Eine Generation meldet sich zu Wort (1. Aufl.). Weinheim: Beltz.

Amato, P. R. (1993). Children's adjustment to divorce. Theories, hypotheses, and empirical support. Journal of Marriage and the Family, 55(1), 23-38.

Amato, P. R. (2000). The consequences of divorce for adults and children. Journal of Marriage and Family, 62(4), 1269-1287.

Amato, P. R. (2010). Research on divorce. Continuing trends and new developments. Journal of Marriage and family, 72(3), 650-666.

Amato, P. R. (2014). The consequences of divorce for adults and children. An update. Društvena istraživanja, 23(1), 5-24.

Amato, P. R., \& Fowler, F. (2002). Parenting practices, child adjustment, and family diversity. Journal of Marriage and Family, 64(3), 703-716.

Amato, P.R., \& Rogers, S. J. (1997). A longitudinal study of marital problems and subsequent divorce. Journal of Marriage and Family, 59(3), 612-624.

Apel, R., \& Kaukinen, C. (2008). On the relationship between family structure and antisocial behavior. Parental cohabitation and blended households. Criminology, 46(1), 35-70.

Baron, R. M., \& Kenny, D. A. (1986). The moderator-mediator variable distinction in social psychological research. Conceptual, strategic, and statistical considerations. Journal of Personality and Social Psychology, 51, 1173-1182.

Bastin, S. (2016). Partnerschaftsverläufe alleinerziehender Mütter. Eine quantitative Untersuchung auf Basis des Beziehungs- und Familienpanels. Dissertation an der Universität Rostock 2014. Wiesbaden: Springer VS.

Ben-Arieh, A., \& Frønes, I. (2011). Taxonomy for child well-being indicators. A framework for the analysis of the well-being of children. Childhood, 18(4), 460-476.

Berger, L. M., \& McLanahan, S.S. (2015). Income, relationship quality, and parenting. Associations with child development in two-parent families. Journal of Marriage and Family, 77(4), 996-1015.

Borrine, M.L., Handal, P. J., Brown, N. Y., \& Searight, H. R. (1991). Family conflict and adolescent adjustment in intact, divorced, and blended families. Journal of Consulting and Clinical Psychology, 59(5), $753-755$.

Broberg, M. (2012). Young children's well-being in Finnish stepfamilies. Early Child Development and Care, 182(3-4), 401-415.

Bronstein, P., Clauson, J., Stoll, M.F., \& Abrams, C.L. (1993). Parenting behavior and children's social, psychological, and academic adjustment in diverse family structures. Family Relations, 42(3), 268-276.

Brown, S. L., Manning, W.D., \& Stykes, J.B. (2015). Family structure and child well-being. Integrating family complexity. Journal of Marriage and Family, 77(1), 177-190.

Bundesministerium für Arbeit und Soziales (2017). Lebenslagen in Deutschland. Der fünfte Armuts- und Reichtumsbericht der Bundesregierung.

Cartwright, C. (2010). Preparing to repartner and live in a stepfamily. An exploratory investigation. Journal of Family Studies, 16(3), 237-250.

Cherlin, A. (1978). Remarriage as an incomplete institution. American Journal of Sociology, 84(3), 634-650.

Coleman, M., Ganong, L., \& Fine, M. (2000). Reinvestigating remarriage. Another decade of progress. Journal of marriage and family, 62(4), 1288-1307.

Conger, R. D., Conger, K. J., \& Martin, M. J. (2010). Socioeconomic status, family processes, and individual development. Journal of Marriage and Family, 72(3), 685-704.

Cummings, E.M., \& Davies, P.T. (2002). Effects of marital conflict on children. Recent advances and emerging themes in process-oriented research. Journal of Child Psychology and Psychiatry, 43(1), 31-63.

Entleitner-Phleps, C., \& Walper, S. (2019). Familienleben nach Trennung und Scheidung: Ein Blick auf komplexe Stieffamilien und kindliches Wohlbefinden in zwei Datensätzen. Vortrag am 10.10.2019 auf der Herbsttagung der Deutschen Gesellschaft für Soziologie/Sektion Familiensoziologie in Bamberg.

Erel, O., \& Burman, B. (1995). Interrelatedness of marital relations and parent-child relations. A metaanalytic review. Psychological Bulletin, 118(1), 108.

Furstenberg, F. F. (2014). Fifty years of family change. From consensus to complexity. Annals of the American Academy of Political and Social Science, 654(1), 12-30. 
Ganong, L. H., \& Coleman, M. (2004). Stepfamily relationships. Development, dynamics, and interventions. New York: Springer.

Ganong, L. H., \& Coleman, M. (1989). Preparing for remarriage. Anticipating the issues, seeking solutions. Family Relations, 38(1), 28-33.

Gennetian, L. A. (2005). One or two parents? Half or step siblings? The effect of family structure on young children's achievement. Journal of Population Economics, 18(3), 415-436.

Ginther, D. K., \& Pollak, R. A. (2004). Family structure and children's educational outcomes. Blended families, stylized facts, and descriptive regressions. Demography, 41(4), 671-696.

Goodman, R. (1997). The strengths and difficulties questionnaire. A research note. Journal of Child Psychology \& Psychiatry \& Allied Disciplines, 38(5), 581-586.

Halpern-Meekin, S., \& Tach, L. (2008). Heterogeneity in two-parent families and adolescent well-being. Journal of Marriage and Family, 70(2), 435-451.

Harold, G. T., \& Sellers, R. (2018). Annual research review. Interparental conflict and youth psychopathology: an evidence review and practice focused update. Journal of Child Psychology and Psychiatry, and Allied Disciplines, 59(4), 374-402.

Hetherington, E. M., \& Jodl, K. M. (1994). Stepfamilies as settings for child development. In A. Booth \& J. Dunn (Hrsg.), Stepfamilies: Who benefits? Who does not? (S. 55-79). London: Routledge.

Hofferth, S.L., \& Anderson, K. G. (2003). Are all dads equal? Biology versus marriage as a basis for paternal investment. Journal of Marriage and Family, 65(1), 213-232.

Hurrelmann, K., Andresen, S., \& Schneekloth, U. (2013). Kinder in Deutschland 2013 (3. World Vision Kinderstudie).

Jurczyk, K., \& Klinkhardt, J. (2014). Vater, Mutter, Kind? Acht Trends in Familien, die Politik heute kennen sollte. Gütersloh: Bertelsmann Stiftung.

King, V., Thorsen, M.L., \& Amato, P.R. (2014). Factors associated with positive relationships between stepfathers and adolescent stepchildren. Social Science Research, 47(0), 16-29.

Kohen, D., \& Guèvremont, A. (2014). Income disparities in preschool outcomes and the role of family, child, and parenting factors. Early Child Development and Care, 184(2), 266-292.

König, W., Lüttinger, P., \& Müller, W. (1987). Eine vergleichende Analyse der Entwicklung und Struktur von Bildungssystemen. Methodologische Grundlagen und Konstruktion einer vergleichbaren Bildungsskala (Comparative Analysis of Social Mobility in Industrial Nations, CASMIN-Projekt, Arbeitspapier Nr. 12). Mannheim.

Kreyenfeld, M., \& Martin, V. (2011). Economic conditions of stepfamilies from a cross-national perspective. Zeitschrift für Familienforschung, 23(2), 128-153.

Lansford, J.E., Ceballo, R., Abbey, A., \& Stewart, A. J. (2001). Does family structure matter? A comparison of adoptive, two-parent biological, single-mother, stepfather, and stepmother households. Journal of Marriage and Family, 63(3), 840-851.

McKee, L., Roland, E., Coffelt, N., Olson, A.L., Forehand, R., Massari, C., Jones, D., Gaffney, C. A., \& Zens, M.S. (2007). Harsh discipline and child problem behaviors. The roles of positive parenting and gender. Journal of Family Violence, 22(4), 187-196.

McMunn, A. M., Nazroo, J. Y., Marmot, M. G., Boreham, R., \& Goodman, R. (2001). Children's emotional and behavioural well-being and the family environment. findings from the Health Survey for England. Social Science \& Medicine, 53(4), 423-440.

Moos, R. H. (1974). The social climate scales. An overview. Palo Alto: Consulting Psychologists.

O'Connor, T. G., Dunn, J., Jenkins, J. M., Pickering, K., \& Rasbash, J. (2001). Family settings and children's adjustment. differential adjustment within and across families. The British Journal of Psychiatry, 179(2), 110-115.

Phillips, T. M. (2012). The influence of family structure vs. family climate on adolescent well-being. Child and Adolescent Social Work Journal, 29(2), 103-110.

Poortman, A.-R. (2005). How work affects divorce. The mediating role of financial and time pressures. Journal of Family Issues, 26(2), 168-195.

Rauschenbach, T., \& Bien, W. (Hrsg.). (2012). Aufwachsen in Deutschland. AID:A - der neue DJI-Survey. Weinheim: Beltz Juventa (eine Veröffentlichung des Deutschen Jugendinstituts e. V. (DJI), München).

Robertson, J. (2008). Stepfathers in families. In J. Pryor (Hrsg.), The international handbook of stepfamilies: Policy and practice in legal, research, and clinical environments (S. 124-150). Hoboken: John Wiley \& Sons Inc.

von Rueden, U., Gosch, A., Rajmil, L., Bisegger, C., \& Ravens-Sieberer, U. (2006). Socioeconomic determinants of health related quality of life in childhood and adolescence. results from a European study. Journal of Epidemiology and Community Health, 60(2), 130-135. 
Simons, R.L., Lorenz, F. O., Conger, R.D., \& Wu, C.-I. (1992). Support from spouse as mediator and moderator of the disruptive influence of economic strain on parenting. Child development, 63(5), 1282-1301.

Statistisches Bundesamt (2018). Deutlich weniger Ehescheidungen im Jahr 2017.

Statistisches Bundesamt (2019). Statistische Wochenberichte Bevölkerung und Arbeit-Monatszahlen 15. $K W / 2019$.

Stewart, S. D. (2005). How the birth of a child affects involvement with stepchildren. Journal of Marriage and family, 67(2), 461-473.

Stewart, S.D. (2006). Brave new stepfamilies. Diverse paths toward stepfamily living. Thousand Oaks: SAGE.

Stocker, C.M., Richmond, M. K., Rhoades, G. K., \& Kiang, L. (2007). Family emotional processes and adolescents' adjustment. Social Development, 16(2), 310-325.

Stormshak, E. A., Bierman, K. L., McMahon, R. J., \& Lengua, L. J. (2000). Parenting practices and child disruptive behavior problems in early elementary school. Journal of Clinical Child Psychology, 29(1), 17.

Sweeney, M. M. (2007). Stepfather families and the emotional well-being of adolescents. Journal of Health and Social Behavior, 48(1), 33-49.

Tillman, K.H. (2008). "Non-traditional" siblings and the academic outcomes of adolescents. Social Science Research, 37(1), 88-108.

Walper, S. (2009). Links of perceived economic deprivation to adolescents' well-being six years later. Zeitschrift für Familienforschung-Journal of Familiy Research, 21(2), 107-127.

Walper, S., Entleitner-Phleps, C., \& Wendt, E.-V. (2016). Brauchen Kinder immer (nur) zwei Eltern? Forschungsergebnisse in Psychologie und Soziologie und ihre Bedeutung für das Kindschaftsrecht. Recht der Jugend und des Bildungswesens, 64(2), 194-210.

Walper, S., Wendt, E.-V., \& Langmeyer, A. (2017). Familiale Sozialisation und Erziehung. In D. Frey \& W. Bierhoff (Hrsg.), Kommunikation, Interaktion und soziale Gruppenprozesse (Enzyklopädie der Psychologie. Themenbereich C Theorie und Forschung. Serie VI Sozialpsychologie, Bd. 3, S. 213-242). Göttingen: Hogrefe. 